TH E

LONDON, EDINBURGH, AND DUBLIN

\title{
PHILOSOPHICAL MAGAZINE
}

\author{
AND \\ JOURNAL OF SCIENCE.
}

[FIFTH SERIES.]

\section{$A P R I L 1887$.}

XXXVI. On the Assumptions necessary for the Theoretical Proof of Arogadro's Law. By Prof. Ludwig Boltzmann (of Graz).*

PROFESSOR TAIT $\dagger$, by making a number of special assumptions, has given a very exact proof of Avogadro's Law-or, rather, of the proposition that in the case of the heat-equilibrium of two mixed gases the mean potential energy of the molecules must be equal. Prof. Tait does not appear to me, however, to have given the least proof that the special assumptions which he makes as the basis of this proposition are necessary, or even that the more general propositions stated by myself $\ddagger$ and Maxwell $\S$ are incorrect.

I believe that in my calculations I have stated precisely the assumptions made, and have invariably drawn the logical conclusions from them, and therefore that I have not deserved the reproach which Prof. Tait makes, that they are "rather of the nature of playing with symbols than of reasoning by consecutive steps," which must also apply to the justmentioned treatise of Maxwell, when he accepts and further develops the propositions of mine upon which Prof. Tait throws doubt. The contradiction of these propositions by certain experimental facts seems to me to have resulted only from too great

* Translated from an advance proof, communicated by the Author, from the Sitzber. d. Wien. Akad. d. Wissensch. vol. xciv, having been read at the Meeting on the 7 th October, 1886.

+ Phil. Mag. [5] vol. xxi. p. 343 (1886).

† Sitzber. d. Wien. Akad. d. Wissensch. rol. Iviii. (1868); vol. Ixiii. March 9 and April 13, 1871; vol. lxvi. October 10, 1872.

$\S$ Camb. Phil. 'Trans. vol. xii. part 13, p. 547 (1879).

Phil. Mag. S. 5. Vol. 23. No. 143. April 1887. 
a generalization thereof. We must remember that analysis can deal only with systems more or less analogous to the molecules of nature, but not with the molecules themselves.

The behaviour of hot bodies is certainly influenced by heatradiation, probably also by movements of electricity \&c.conditions which bave not been taken into account by me, nor in any other mechanical theory of heat. Absolute agreement with facts cannot therefore be expected. It is therefore only possible to consider (1) whether the propositions stated really follow logically from the assumptions made, and (2) whether the analogy between the properties of the system considered and those of hot bodies undoubtedly exists.

This analogy can only be made perfectly clear by reference to all my papers, and in particular to those on the second law of Thermodynamics.

Although Mr. Burbury* has made well-founded objections to the propositions of Prof. Tait, it appears to me desirable to discuss still more rigorously the question, Which of Prof. Tait's assumptions are really necessary to the proof? In doing this I will follow Prof. Tait's method, and at first will treat only some special cases, in order not to become unintelligible by too great a generalization. Although he does not expressly say so, Prof. Tait yet implicitly assumes that two molecules upon impact behave like elastic spheres. For under any other law of mutual action Prof. Tait's equations on p. 346 would only hold good in case the quantities which he denotes by $u$ and $\mathrm{v}$ were the components in the direction of the apsides ; and the calculations on p. 347 would then not be applicable to these equations. I will make the same assumption, and use the notation of my Theory of Gaseous Diffusion, part i. $†$

I have there treated the impact of two elastic balls as generally as possible with reference to the Theory of Gases. The figures and formulæ are certainly a little copions, but are generally applicable, and, as I believe, also clear, when once their meaning has been comprehended.

Let two elastic spheres (molecules) of masses $m$ and $\mathrm{M}$ impinge upon each other. Let $v=\Omega v$ and $\nabla=\Omega V$ be their velocities before impact (see fig. 1), and $v^{\prime}=\Omega v^{\prime}$ and $V^{\prime}=\Omega V^{\prime}$ their velocities after impact, $\delta$ the sum of radii of the spheres, $r=v \mathrm{~V}$ their relative velocity before impact, and suppose their relative velocity after impact to have the direction $r^{\prime}=v^{\prime} V^{\prime}$. Let $\Omega \mathrm{C}$ be the line of centre of the spheres at the moment of impact.

* Phil. Mag. [5] vol. xxi. p. 481 (1886).

$\dagger$ Sitzber. d. Wien. Akad. d. Wissensch. vol. lxxxvi. p. 63 (June 1882). 
for the Theoretical Proof of Avogadro's Law.

Let $\nless v \mathrm{~V}=\mathrm{T}, \quad \nless v r=\mathrm{G}, \quad \nless r r^{\prime}=2 \mathrm{~S}, \quad \nless r, \Omega \mathrm{C}=90^{\circ}+\mathrm{S}$, $\$ v^{\prime} \mathrm{V}^{\prime}=\mathrm{T}^{\prime}, \quad \nless v^{\prime} r^{\prime}=\mathrm{G}^{\prime}$.

Fig. 1.

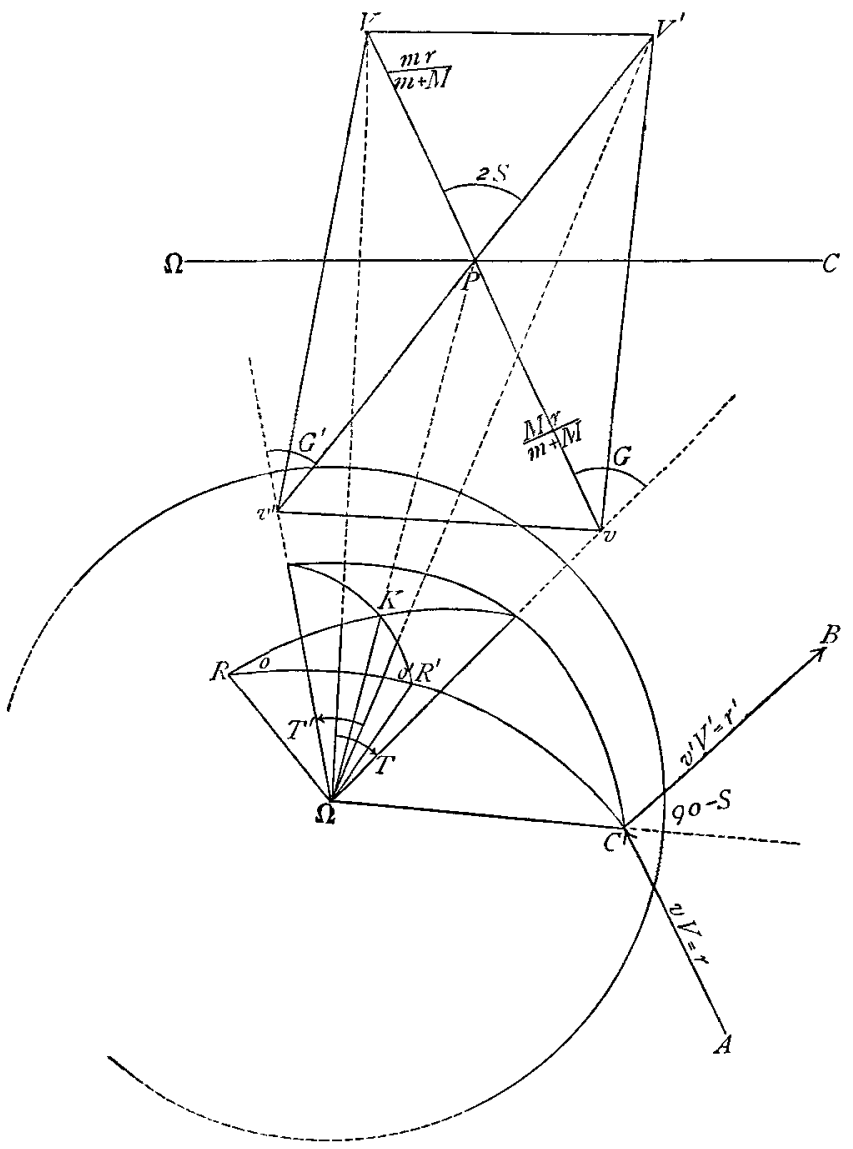

Let $O$ be the angle of the planes $r, v$, and $r, \Omega \mathrm{C}$; and $O^{\prime}$ the angle of the planes $r^{\prime}, v^{\prime}$, and $r^{\prime}, \Omega \mathrm{C}$. I denote the sines and cosines of the angles by the corresponding Roman and Greek letters. For the sake of clearness I include in fig. 1 those lines of fig. 2 of my Theory of Gas Diffusion, just mentioned, which are here necessary. Then, according to formula (21) of p. 72 of that treatise,

$$
\mathrm{D}=\frac{m v^{\prime 2}}{2}-\frac{m v^{2}}{2}=\frac{2 m \mathrm{M} v r}{m+\mathrm{M}}\left(g \sigma^{2}-\gamma \delta \sigma o\right)+\frac{2 m \mathrm{M}^{2} r^{2} \sigma^{2}}{(m+\mathrm{M})^{2}} .
$$


The right-hand side agrees completely with the expression which Prof. Tait finds on p. 346 for $\frac{P}{2}\left(u^{\prime 2}-u^{2}\right)$, since, according to equation (20) of my treatise, the quantity which Prof. Tait denotes by $\mathrm{u}$ has the value $v(-g \sigma+\gamma s o)$, where $\mathrm{u}-\mathrm{v}$ is the projection of $r$ on $\Omega \mathrm{C}$, which is equal to $r \sigma$. Now let there be very many molecules of mass $m$ (molecules of the first kind), as also of those of mass $M$ (molecules of the second kind), uniformly mixed in a space and uniformly distributive with reference to the directions of their velocities. Of all possible impacts which in general may occur, we will first select only those for which the variables $v, \mathrm{~V}, \mathrm{~T}, \mathrm{~S}, \mathrm{O}$ lie between the infinitely close limits,

$$
\left.\begin{array}{c}
v \text { and } v+d v, \mathrm{~V} \text { and } \mathrm{V}+d \mathrm{~V}, \mathrm{~T} \text { and } \mathrm{T}+d \mathrm{~T}, \\
\mathrm{~S} \text { and } \mathrm{S}+d \mathrm{~S}, \quad \mathrm{O} \text { and } \mathrm{O}+d \mathrm{O} .
\end{array}\right\} .
$$

In fig 1 a sphere of radius $S$, supposed concentric with one of the molecules of mass M, is drawn. All straight lines are drawn dotted from their intersections with the surface of this sphere. The arcs are arcs of great circles of this sphere. The molecules of mass $m$ fly against this sphere, so that their centres describe the straight line $A C$ relative to the first molecule which is parallel to $v \mathrm{~V}$ and $\Omega R$. At the point $\mathrm{C}$ the centres are reflected in the direction $\mathrm{CB}$, which is parallel to $v^{\prime} \mathrm{V}$ and $\Omega \mathrm{R}^{\prime}$. The relative motion before impact, with which alone we are concerned, remains unaltered if we imagine the molecules of mass $m$ at rest and the sphere drawn in fig. 1 moving with the velocity $r=v \mathrm{~V}$ in direction opposite to $\Omega \mathrm{R}$. If its surface be divided by a plane passing through its centre at right angles to $\Omega \mathrm{R}$, then, with our last-mentioned conception of relative motion, the preceding half-sphere would, in unit time, pass through a space bounded by two half-spheres and a cylindrical surface of volume $\pi \delta^{2} r$. Of this whole space a small portion is now to be cut out, as follows :- $\Omega \mathrm{C}$ is that radius of the sphere which is parallel to the direction of the line of centres. Whilst the point $\mathrm{C}$ is so moved on the surface of the sphere that $S$ increases by the amount $d S$ without change of direction of $\Omega R, C$ describes a linear element of length $\delta d \mathrm{~S}$. If, on the other hand, we move $\mathrm{C}$ so that $\mathrm{O}$ increases by $d O, \mathrm{C}$ describes a linear element of length $\delta s d O$. These two determine an element of area on the surface of the sphere of area $\delta^{2} s d \mathrm{~S} d 0$. This is inclined at an angle $\mathrm{S}$ to the direction of $r$. Since the half-sphere moves with the velocity $r$ in the direction $-\Omega \mathrm{R}$, this element of surface moves through a prism of volume $r \delta^{2} s \sigma d \mathrm{~S} d 0$. So soon as the centre of a 
molecule of the first kind lies within this prism, it impinges in the manner described upon a molecule of the second kind.

Of all molecules of the first kind lying within this prism, we have to consider only those whose velocities lie between the limits $v$ and $v+d v$, and which are moving in directions making angles with the direction of $\mathrm{V}$ which lie between the limits $\mathrm{T}$ and $\mathrm{T}+d \mathrm{~T}$. If there are in the unit volume $4 \pi v^{2} f(v) d v$ molecules of the first kind which fulfil the first condition, then, in the above-described prism, there are

$$
2 \pi v^{2} f(v) r \delta^{2} \tau s \sigma . d v d \mathrm{~S} d \mathrm{~T} d \mathrm{O}
$$

molecules of the first kind which also satisfy the second condition.

Let us further suppose that in the unit volume there are $4 \pi V^{2} F(V) d V$ molecules of the second kind whose velocities lie between the limits $\mathrm{V}$ and $\mathrm{V}+d \mathrm{~V}$. Then, by multiplying the above expression by this factor, the following expression for the total number of impacts which occur in unit time in unit volume between a molecule of the first kind and a molecule of the second kind, so that the conditions (2) are fulfilled,

$$
d \mathrm{Z}=8 \pi^{2} v^{2} \mathrm{~V}^{2} f(v) \mathrm{F}(\mathrm{V}) r \delta^{2} \tau s \sigma d v d \mathrm{~V} d \mathrm{~T} d \mathrm{~S} d \mathrm{O}, .
$$

is obtained.

In order to obtain from this the number $\mathrm{Z}$ of all the impacts which may occur altogether in the unit volume in unit time between a molecule of the first kind and one of the second kind, we have to integrate with reference to $\mathrm{O}$ from zero to $2 \pi$, with reference to $\mathrm{T}$ from zero to $\pi$, with reference to $\mathrm{S}$ from zero to $\frac{\pi}{2}$, and with reference to $v$ and $V$ from zero to $\infty$; which we may express by $Z=\int d Z$.

$\mathrm{J}=\int \mathrm{D} d \mathrm{Z}$ is the energy which is brought by all these impacts to molecules of the first kind. If we integrate the expression $\mathrm{D} d \mathrm{Z}$ first with reference to $\mathrm{O}$ and $\mathrm{S}$, we obtain

$$
8 \pi^{3} v^{2} f(v) \cdot \mathrm{V}^{2} \mathrm{~F}(\mathrm{~V}) r \delta^{2} \tau d v d \mathrm{~V} d \mathrm{~T} \frac{m \mathrm{M}}{m+\mathrm{M}}\left(v r g+\frac{\mathrm{M} r^{2}}{\mathrm{M}+m}\right)
$$

whilst $d Z$ integrated with reference to the same variables gives

$$
8 \pi^{3} v^{2} f(v) \mathrm{V}^{2} \mathrm{~F}(\mathrm{~V}) r \delta^{2} \tau d v d \mathrm{~V} d \mathrm{~T} .
$$

The quotient of the two expressions may be considered to be the energy which, on the average, is transferred from molecules of the first kind to molecules of the second kind, when 
the magnitudes of the velocities $v$ and $\mathrm{V}$ and their angle $\mathrm{T}$ are given. This quotient is

$$
\frac{m \mathrm{M}}{m+\mathrm{M}}\left[v r g+\frac{\mathrm{M} r^{2}}{m+\mathrm{M}}\right] \text {. }
$$

This agrees exactly with the first expression for $l_{1}^{\prime}-l_{1}$, which Stefan* finds in his treatise on the dynamical theory of the diffusion of gases towards the end of the second part, and which, after correction of some printer's errors, is as follows :-

$$
l_{1}^{\prime}-l_{1}=-\frac{2 m m_{1}}{\left(m_{1}+m_{2}\right)^{2}}\left[l_{1}-l_{2}-\frac{m_{1}-m_{2}}{2}\left(x_{1} x_{2}+y_{1} y_{2}+z_{1} z_{2}\right)\right] ;
$$

for, in Stefan's formula,

$$
l_{1}=m \frac{v^{2}}{2}, \quad l_{2}=\mathbb{M} \frac{\mathrm{V}^{2}}{2}, \quad x_{1} x_{2}+y_{1} y_{2}+z_{1} z_{2}=v \mathrm{~V} t=v r g+v^{2},
$$

and

$$
\mathrm{V}^{2}=v^{2}+r^{2}+2 v r g
$$

But the first conclusions of Stefan's would apply only where the molecules act upon each other with a force inversely proportional to the fifth power of the distance. In this case the factor $r$ disappears from the expression $d Z$, and on further integration in respect of $d \mathrm{~T}$ the terms

disappear.

$$
x_{1} x_{2}+y_{1} y_{2}+z_{1} z_{2}
$$

With elastic spheres, on the other hand, the impacts are so much the more probable the greater the relative velocity $r$; therefore, for each of the products $x_{1} x_{2}, y_{1} y_{2}, z_{1} z_{2}$, positive values are more probable than negative values, and we cannot, therefore suppose

$$
x_{1} x_{2}+y_{1} y_{2}+z_{1} z_{2}=0
$$

on the average. In fact, if we wish also to integrate expresssion (4) with reference to $\mathrm{T}$, we must observe that

$$
r^{2}=v^{2}+\nabla^{2}-2 v V t \text { and } r g=V t-v \text {. }
$$

Integration gives, therefore,

$$
\frac{8 \pi^{3} m \mathrm{M}}{m+\mathrm{M}} v f(v) \mathrm{VF}(\mathrm{V}) \delta^{2} d v d V \int\left(\frac{\mathrm{V}^{2}-v^{2}}{2}+\frac{\mathrm{M}-m}{\mathrm{M}+m} \frac{r^{2}}{2}\right) r^{2} d r \text {; }
$$

the upper limit is $\mathrm{V}+v$, the lower $\mathrm{V}-v$ or $v-\mathrm{V}$ according as $V>v$ or the reverse. In the first case the integral has the form

$$
-\frac{1}{3} v^{5}-\frac{2}{3} v^{3} \mathrm{~V}^{2}+v \mathrm{~V}^{4}+\frac{\mathrm{M}-m}{\mathrm{M}+m}\left(\frac{\mathrm{l}}{5} v^{5}+2 v^{3} \mathrm{~V}^{2}+v \mathrm{~V}^{4}\right) .
$$

* Sitzber. d. Wien. Akad. d. Wissensch. vol. lxv. April 1872. 
In the latter case $\mathrm{V}$ and $v$ must be exchanged. If, therefore, the functions $f$ and $\mathbf{F}$ are chosen quite arbitrarily, $\int \mathrm{D} d \mathrm{Z}$ will in general not disappear if the molecules of the first and second kinds have equal energy. In other words, even though the molecules of the first and second kinds have equal energy, yet, if $f$ and $F$ are chosen arbitrarily, energy may at first be transferred from molecules of the first kind to molecules of the second kind, or vice versâ. It would be transferred to them continuously if by any external action the originally chosen values of the functions $f$ and $\mathrm{F}$ were maintained constant.

If, for example, with uniform distribution in space and equal probability of all directions of velocity, the molecules of the first kind had all the same velocity $v$, the second kind all the same velocity $\mathrm{V}>v$, then there would be at first no transference of energy to the "molecules of the first kind by the molecules of the second kind if expression (5) should disappear. If we put the positive quantities

we have

$$
\frac{m-\mathrm{M}}{m+\mathrm{M}}=\mu, \quad \frac{\mathrm{V}^{2}}{v^{2}}=x,
$$

$$
\begin{gathered}
(1-\mu) x=\frac{1}{3}+\mu+\sqrt{\frac{4}{9}+\frac{8 \mu}{15}+\frac{4 \mu^{2}}{5}}, \\
3 \mathrm{M} x=2 m-\mathrm{M}+\sqrt{\frac{4}{5}\left(5 m^{2}-2 m \mathrm{M}+2 \mathrm{M}^{2}\right)} .
\end{gathered}
$$

If $m$ is only a little larger than $M$, say $m=M(1+\epsilon)$, then this gives nearly

for $m=4 \mathrm{M}$, we have

$$
\mathrm{MV}^{2}=m v^{2} \cdot\left(1+\frac{\epsilon}{5}\right) ;
$$

$$
M V^{2}=m v^{2}(1 \cdot 21 \ldots) .
$$

If $m$ is very much greater than $\mathrm{M}$, we have nearly

$$
\mathrm{MV}^{2}=\frac{4}{3} m v^{2} \text {. }
$$

The condition that, on the average, no energy shall be communicated in the first moment to the molecules of the first order, requires then that the molecules of smaller mass should bave greater energy than the others. But it must be observed that this only holds good for the first moment; the velocities even of the molecules of the first kind immediately become different amongst themselves as the result of impacts, and so 
also those of the molecules of the second order; so that the conditions at once become completely altered.

If we assume Maxwell's law of distribution of velocities, by putting $f(v)=\mathrm{A} e^{-a z^{2}}, \mathrm{~F}(\mathrm{~V})=\mathrm{B} e^{-b \mathrm{~V}^{2}}$ we find, by precisely the same calculation as that of Prof. Tait, that there is no exchange of energy between the molecules of the first kind and those of the second, if the mean energy of both is the same-which, (following Prof. Tait) we will call Maxwell's Theorem.

So far all Prof. Tait's conclusions are, without doubt, correct. But it does not yet follow from this that the existence of Maxwell's law of distribution must be assumed in order to prove Maxwell's theorem. It may rather be shown that, whatever the masses and ratio of diameters may be, if only the molecules of the first order come generally into collision with those of the second order, then Maxwell's distribution of velocities is spontaneously brought about both amongst the former and also amongst the latter molecules.

In this it is not even necessary to assume that the molecules of the first order are generally in collision amongst themselves, nor that the molecules of the second order are in collision amongst themselves. The only assumptions are : that both the molecules of the first and also those of the second order are uniformly distributed over the whole space; that throughout they behave in the same way in all directions; and that the duration of the impact is short in comparison with the time between two impacts. I have given* the proof of this in my paper on the Thermal Equilibrium of Gases acted on by External Forces, at the conclusion of $\S 1$; but as I have there only briefly indicated the mode of calculation and have only given the result, Prof. Tait has probably quite overlooked the passage, and I will be more explicit on the present occasion.

Let the molecules of the first and second kinds of gas be at the beginning of the time $(t=0)$ uniformly distributed in a space enclosed by rigid perfectly elastic walls; but let the distribution of energy among them be altogether arbitrary. Exactly as before, let

$$
4 \pi v^{2} f(v, o) d v
$$

be the number of molecules of the first kind of gas in the unit volume whose velocities lie between the limits

$$
v \text { and } v+d v \text {. . . . . . . }
$$

In exactly the same way, let $4 \pi \mathrm{V}^{2} \mathrm{~F}(\mathrm{~V}, 0) d \mathrm{~V}$ be the number of molecules whose velocities lie between

$$
\mathrm{V} \text { and } \mathrm{V}+d \mathrm{~V} \text {. . . . . . }
$$

* Wien. Sitzb. vol. lxxii., October 1875. 
Since no direction in space, and also no element of volume, has any advantage over another, we may assume that the distribution of energy also remains uniform for all succeeding time. But in general this is prevented by the collisions which occur. At the time $t$, let there be in the unit volume $4 \pi v^{2} f^{\prime}(v, t) d v$ molecules of the first kind of gas whose velocities lie between the limits $(6), \mathrm{F}(v, t)$ having a similar signification.

Evidently the problem is conceived in its utmost generality if we imagine $f(v, o)$ and $\mathrm{F}(\mathrm{V}, o)$ having any given values, and determine the changes of these functions in course of time. Evidently we have first of all to determine the increase

$$
\theta \frac{\partial f(v, t)}{\partial t} \text { and } \theta \frac{\partial \mathrm{F}(\mathrm{V}, t)}{\partial t}
$$

which the functions $f$ and $\mathrm{F}$ undergo, whilst the time increases from $t$ to $t+\theta$. During the element of time $\theta$, let $n$ molecules in the unit volume out of the $4 \pi v^{2} f(v, t) d v$ molecules of the first kind therein contained, whose energies lie between the limits (6), enter into collision with other molecules of the first kind, and $\mathrm{N}$ molecules with those of the second kind. Let us imagine $\theta$ so chosen that $n$ and $\mathrm{N}$, although large numbers, are yet small in comparison with $4 \pi v^{2} f(v, t) d v$.

Since the number of those molecules for which the velocities after impact also lie between the limits (6), or for which the velocity of the second impinging molecule lies between the same limits, is of an order higher only by an infinitely small amount, we may assume that the velocities of all these $n$ molecules and also of the $\mathrm{N}$ molecules after lapse of the time $\theta$, no longer lie between the limits (b). $n+\mathrm{N}$ is therefore the number of those molecules whose velocities were at the beginning of the time $\theta$ between those limits, but at the end of that time were not between the same limits.

But during the time $\theta$, other molecules whose velocities were not previously between the limits (6), in consequence of impacts acquire a velocity lying between these limits. Let, then, $p$ molecules of the first kind acquire a velocity lying between these limits by impacts with other molecules of the first kind, and $\mathrm{P}$ molecules of the first kind by impacts with molecules of the second kind. Then

$$
4 \pi v^{2} \theta \frac{\partial f(v, t)}{\partial t}=p+\mathrm{P}-n-\mathrm{N} . \quad . \quad .
$$

We found before the expression (3) for the number of impacts which in unit time and unit volume so occur that the variables $v, \mathrm{~V}, \mathrm{~T}, \mathrm{~S}$, and $\mathrm{O}$ lie between the limits (2). 
Integrating this expression for all the other differentials up to $d v$ and multiplying by $\theta$, we have

$\mathrm{N}=8 \pi^{2} v^{2} f(v, t) d v \delta^{2} \theta \int_{0}^{\infty} \int_{0}^{\pi} \int_{0}^{\frac{\pi}{2}} \int_{0}^{2 \pi} \mathrm{V}^{2} \mathrm{~F}(\mathrm{~V}, t) r \tau s \sigma d \mathrm{~V} d \mathrm{~T} d \mathrm{~S} d \mathrm{O}$.

The kind and mode of impact is completely determined by the values of the variables $v, V, T, S$, and $O$. The magnitudes $v^{\prime}$ and $V^{\prime}$ of the velocities of both molecules after impact, their angle $T^{\prime}$ as well as the angle $O^{\prime}$ which the plane $R \Omega R^{\prime}$ of the two relative velocities makes with the plane $v^{\prime} \Omega^{\prime} \nabla^{\prime}$ of the two absolute velocities $v^{\prime}$ and $V^{\prime}$ after the impact, may therefore be expressed as functions of $v, \mathrm{~V}, \mathrm{~T}, \mathrm{~S}$, and $\mathrm{O}$. For all impacts for which the latter variables lie between the limits (2), $v^{\prime}, \mathrm{V}^{\prime}, \mathrm{T}^{\prime}, \mathrm{S}^{\prime}$, and $\mathrm{O}^{\prime}$ will also lie between certain infinitely close limits, which we may denote by

$$
\begin{gathered}
v^{\prime} \text { and } v^{\prime}+d v^{\prime}, \quad \mathrm{V}^{\prime} \text { and } \mathrm{V}^{\prime}+d \mathrm{~V}^{\prime}, \\
\mathrm{T}^{\prime} \text { and } \mathrm{T}^{\prime}+d \mathrm{~T}^{\prime}, \quad \mathrm{O}^{\prime} \text { and } \mathrm{O}^{\prime}+d \mathrm{O}^{\prime}, \quad \mathrm{S} \text { and } \mathrm{S}+d \mathrm{~S} .
\end{gathered}
$$

The angle $\mathrm{S}$ has the same meaning before and after impact, and is therefore after impact also included within the limits $\mathbf{S}$ and $\mathrm{S}+d \mathrm{~S}$. But it is now clear that each impact may also be taken in inverted order. If, therefore, inversely the values of the variables before impact lay between the limits (10), then they would also after impact lie between the limits (2). Exactly as with expression (3), so also will

$$
d \mathrm{Z}^{\prime}=8 \pi^{2} v^{\prime 2} \mathrm{~V}^{\prime 2} f\left(v^{\prime}, t\right) \mathrm{F}\left(\mathrm{V}^{\prime}, t\right) \theta r \delta^{2} \tau^{\prime} s \sigma d v^{\prime} d \mathrm{~V}^{\prime} d \mathrm{~T}^{\prime} d \mathrm{O}^{\prime} d \mathrm{~S}^{\prime}
$$

be the number of impacts which, in the unit volume during the time $\theta$, occur between a molecule of the first order and one of the second, so that after impact the variables shall lie between the limits (2). Since $v^{\prime}, V^{\prime}, T^{\prime}$, and $O^{\prime}$ are known as functions of $v, \mathrm{~V}, \mathrm{~T}, \mathrm{O}$, and $\mathrm{S}$, we may here again introduce the differentials of the latter variables, and obtain

$$
d \mathrm{Z}^{\prime}=8 \pi^{2}{v^{\prime 2}}^{\prime 2} f\left(v^{\prime}, t\right) \mathbf{F}\left(\mathrm{V}^{\prime}, t\right) \theta r \delta^{2} \tau^{\prime} s \sigma \Delta d v d \mathrm{~V} d \mathrm{~T} d \mathrm{O} d \mathrm{~S},
$$

where

$$
\Delta=\Sigma \pm \frac{\partial v^{\prime}}{\partial v} \cdot \frac{\partial V^{\prime}}{\partial V} \cdot \frac{\partial T^{\prime}}{\partial T} \cdot \frac{\partial O^{\prime}}{\partial O}, \ldots . . .
$$

the partial differentials being taken under the supposition that $\mathrm{S}$ is constant. If we integrate expression (12) for all variables except $d v$, we obtain all impacts which occur in unit volume during the time $\theta$ between a molecule of the first and a molecule of the second kind, so that after impact the velocities of the molecules of the first kind lie between the limits (6). 
for the Theoretical Proof of Avogadro's Law.

$\mathrm{P}=8 \pi^{2} \theta \delta^{2} d v \int_{0}^{\infty} \int_{0}^{\pi} \int_{0}^{\pi} \int_{0}^{2 \pi} v^{\prime 2} \mathrm{~V}^{\prime 2} f\left(v^{\prime}, t\right) \mathrm{F}\left(\mathrm{V}^{\prime}, t\right) r \tau^{\prime} s \sigma \Delta d \mathrm{~V} d \mathrm{~T} d \mathrm{~S} d \mathrm{O}$

Maxwell* has already given an equation which in our notation is

$$
\Delta v^{\prime 2} V^{\prime 2} \tau^{\prime}=v^{2} V^{2} \tau \text {. . . . . . . }
$$

He has, it is true, indicated a proof of this equation, but has not clearly shown its truth.

I have proved this proposition more fully, and obtained a similar one for polyatomic molecules; on which account I have also assumed it in my treatise on the thermal equilibrium of gases upon which external forces act. The most general proposition, in which all similar propositions are included, is shown in Maxwell's paper "On Boltzmann's Theorem." $\dagger$ But since all the necessary formulæ are now at our disposal, I will here verify equation (15) by direct calculation of the functional determinants.

If we first of all introduce, instead of $\mathrm{V}$ and $\mathrm{T}$, the variables $r$ and $G$, we obtain $V^{2} d V \tau d T=r^{2} d r \gamma d G$, as is geometrically evident. So also

$$
\nabla^{\prime 2} d V^{\prime} \tau^{\prime} d \mathrm{~T}^{\prime}=r^{2} d r \gamma^{\prime} d G^{\prime}
$$

Since $r$ is not altered by impact, we introduce, instead of $d v$, $d \mathrm{G}, d \mathrm{O}$, the differentials of the three variables,

$$
\begin{aligned}
& x=v^{2}=v^{2}+\frac{4 M v r}{m+M}\left(g \sigma^{2}-\gamma s \sigma o\right)+\frac{4 M^{2} r^{2} \sigma^{2}}{(m+M)^{2}}, \\
& y=(m+\mathrm{M}) v^{2}+2 \mathrm{M} v r g+\mathrm{M} r^{2}, \\
& z=v \gamma \omega .
\end{aligned}
$$

The calculation of the functional determinants gives $d x d y d z=\frac{4 \mathrm{M} v^{2} r \gamma}{m+\mathrm{M}}\left[(m+\mathrm{M}) v\left(2 g s \sigma-\gamma s^{2} o+\gamma \sigma^{2} o\right)+2 \mathrm{M} r s \sigma\right] d v d \mathrm{G} d 0$.

Further, from the spherical triangle RKR' (fig. 1), we have

$$
\omega: \omega^{\prime}=\sin R^{\prime} \Omega K: \sin R \Omega K \text {; }
$$

and from the triangles $\mathrm{P} \Omega v$ and $\mathrm{P} \Omega v^{\prime}$,

$$
\begin{aligned}
& \sin \mathrm{R} \Omega \mathrm{K}: \gamma=v: \Omega \mathrm{P}, \\
& \sin \mathrm{R}^{\prime} \Omega \mathrm{K}: \gamma^{\prime}=v^{\prime}: \Omega \mathrm{P} ;
\end{aligned}
$$

* Phil. Mag. [4] vol. xxxv. (March 1868).

† Cambridge Phil. Trans. vol. xii. part 3, p. 547 (1879). Wied. Beibl. v. p. $403(1881)$. 
316 Prof. L. Boltzmann on the Assumptions necessary therefore

whence

$$
v \gamma \omega=v^{\prime} \gamma^{\prime} \omega^{\prime}=z ; . . . \quad . \quad .
$$

$\left(v^{\prime} \gamma^{\prime} o^{\prime}\right)^{2}=v^{\prime 2}-v^{\prime 2} g^{\prime 2}-v^{2} \gamma^{2} \omega^{2}=\left\{v\left(2 g s \sigma-\gamma^{2} o+\gamma \sigma^{2} o\right)+\frac{2 \mathrm{M} r \sigma s}{n+\mathrm{M}}\right\}^{2}$.

Therefore

Since, further,

$$
d x d y d z=4 \mathrm{M} v^{2} v^{\prime} r \gamma \gamma^{\prime} o^{\prime} d v d \mathrm{G} d \mathrm{O} .
$$

$$
x=v^{\prime 2}, y=(m+\mathrm{M}) v^{\prime 2}+2 \mathrm{M} v^{\prime} r g^{\prime}+\mathrm{M} r^{2}, z=v^{\prime} \gamma^{\prime} \omega^{\prime},
$$

it follows that

$$
d x d y d z=4 \mathrm{M} v^{\prime 3} r \gamma^{\prime 2} o^{\prime} d v^{\prime} d \mathrm{G}^{\prime} d \mathrm{O}^{\prime} ;
$$

whence the relationship to be proved between $d v d \mathrm{G} d \mathrm{O}$ and $d v^{\prime} d \mathrm{G}^{\prime} d \mathrm{O}^{\prime}$ follows. But, according to equation (15), it follows from (14) that

$\mathrm{P}=8 \pi^{2} \delta^{2} \theta d v \int_{0}^{\infty} \int_{0}^{\pi} \int_{0}^{\frac{\pi}{2}} \int_{0}^{2 \pi} v^{2} \mathrm{~V}^{2} f\left(v^{\prime}, t\right) \mathrm{F}\left(\mathrm{V}^{\prime}, t\right) r \tau s \sigma d \mathrm{~V} d \mathrm{~T} d \mathrm{~S} d \mathrm{O}$.

$n$ and $p$ can be found from $\mathrm{N}$ and $\mathrm{P}$ by simple exchange of the function $\mathrm{F}$ and the mass $M$ for $f$ and $m$, and in place of $\delta$ we have $\lambda$ the diameter of a molecule of the first kind.

Hence

$$
\left.\begin{array}{l}
n=8 \pi^{2} v^{2} f(v, t) d v \lambda^{2} \theta \int_{0}^{\infty} \int_{0}^{\pi} \int_{0}^{\frac{\pi}{2}} \int_{0}^{2 \pi} \mathrm{V}^{2} f(\mathrm{~V}, t) r \tau s \sigma d \mathrm{~V} d \mathrm{~T} d \mathrm{~S} d \mathrm{O}, \\
p=8 \pi^{2} v^{2} d v \lambda^{2} \theta \int_{0}^{\infty} \int_{0}^{\pi} \int_{0}^{\frac{\pi}{2}} \int_{0}^{2 \pi} \mathrm{V}^{2} f\left(v^{\prime}, t\right) f\left(\mathrm{~V}^{\prime}, t\right) r \tau s \sigma d \mathrm{~V} d \mathrm{~T} d \mathrm{~S} d \mathrm{O},
\end{array}\right\}
$$

and thence

$$
\left.\begin{array}{r}
\frac{\partial f(v, t)}{\partial t}=2 \pi \lambda^{2} \int_{0}^{\infty} \int_{0}^{\pi} \int_{0}^{\frac{\pi}{2}} \int_{0}^{2 \pi}\left(f^{\prime} f_{1}-f f_{1}\right) \mathrm{V}^{2} r \tau s \sigma d \mathrm{~V} d \mathrm{~T} d \mathrm{~S} d \mathrm{O} \\
+2 \pi \delta^{2} \int_{0}^{\infty} \int_{0}^{\pi} \int_{0}^{\frac{\pi}{2}} \int_{0}^{2 \pi}\left(f^{\prime} \mathrm{F}_{1}^{\prime}-f \mathrm{~F}_{1}\right) \mathrm{V}^{2} r \tau s \sigma d \mathrm{~V} d \mathrm{~T} d \mathrm{~S} d \mathrm{O},
\end{array}\right\}
$$


In this $f=f(v, t), f_{1}=f(\mathrm{~V}, t), f^{\prime}=f\left(v^{\prime}, t\right), f_{1}^{\prime}=f\left(\mathrm{~V}^{\prime}, t\right)$, $\mathrm{F}=\mathrm{F}(v, t), \mathrm{F}^{\prime}=\mathrm{F}\left(v^{\prime}, t\right), \mathrm{F}_{1}=\mathrm{F}(\mathrm{V}, t), \mathrm{F}_{1}^{\prime}=\mathrm{F}\left(\mathrm{V}^{\prime}, t\right) . \quad \Lambda$ is the diameter of a molecule of the second kind.

By means of these equations we can show that the quantity

$$
\mathrm{E}=\int_{0}^{\infty} f(l f-1) v^{2} d v+\int_{0}^{\infty} \mathrm{F}_{1}\left(l \mathrm{~F}_{1}-1\right) \mathrm{V}^{2} d \mathrm{~V} .
$$

(whose intimate connection with the magnitude called by Clausius "Entropy" I have remarked elsewhere) can only decrease or (in an extreme case) remain constant. $l$ denotes the natural logarithm. We have

$$
\frac{d \mathrm{E}}{d t}=\int_{0}^{\infty} l f \frac{\partial f}{\partial t} v^{2} d v+\int_{0}^{\infty} l f \mathrm{~F}_{1} \frac{\partial \mathrm{F}_{1}}{\partial t} \mathrm{~V}^{2} d \mathrm{~V} ;
$$

therefore, with reference to the equations (20),

$$
\left.\begin{array}{c}
\frac{1}{2 \pi} \frac{\partial \mathrm{E}}{\partial t} \\
=\int_{0}^{\infty} \int_{0}^{\infty} \int_{0}^{\pi} \int_{0}^{\frac{\pi}{2}} \int_{0}^{2 \pi} \lambda^{2} l f .\left(f^{\prime} f_{1}^{\prime}-f f_{1}\right) v^{2} \mathrm{~V}^{2} r \tau s \sigma d v d \mathrm{~V} d \mathrm{~T} d \mathrm{~S} d \mathrm{O} \\
+\int_{0}^{\infty} \int_{0}^{\infty} \int_{0}^{\pi} \int_{0}^{\frac{\pi}{2}} \int_{0}^{2 \pi} \Lambda^{2} l \mathrm{~F}_{1}\left(\mathrm{~F}^{\prime} \mathrm{F}_{1}^{\prime}-\mathrm{FF}_{1}\right) v^{2} \mathrm{~V}^{2} r \tau s \sigma d v d \mathrm{~V} d \mathrm{~T} d \mathrm{~S} d \mathrm{O} \\
+\int_{0}^{\infty} \int_{0}^{\infty} \int_{0}^{\pi} \int_{0}^{\frac{\pi}{2}} \int_{0}^{2 \pi} \delta^{2} l f\left(f^{\prime} \mathrm{F}_{1}^{\prime}-f \mathrm{~F}_{1}\right) v^{2} \mathrm{~V}^{2} r \tau s \sigma d v d \mathrm{~V} d \mathrm{~T} d \mathrm{~S} d \mathrm{O} \\
+\int_{0}^{\infty} \int_{0}^{\infty} \int_{0}^{\pi} \int_{0}^{\frac{\pi}{2}} \int_{0}^{2 \pi} \delta^{2} l \mathrm{~F}_{1}\left(f^{\prime} \mathrm{F}_{1}^{\prime}-f \mathrm{~F}_{1}\right) v^{2} \mathrm{~V}^{2} r \tau s \sigma d v d \mathrm{~V} d \mathrm{~T} d \mathrm{~S} d \mathrm{O}
\end{array}\right\}
$$

From the circumstance that every impact may take place also in inverted order, it follows at once that the value of any definite integral which, according to the above, is to be extended over all possible values of the variables before impact, does not alter if we exchange the value of the variables before impact for their values after impact, and vice versâ, and, finally, again integrate for all possible values.

We shall have, therefore, for each function $\Psi$ compounded in any way of the variables under the functional sign, 
318 Prof. L. Boltzmann on the Assumptions necessary

$$
\begin{gathered}
\int_{0}^{\infty} \int_{0}^{\infty} \int_{0}^{\pi} \int_{0}^{\frac{\pi}{2}} \int_{0}^{2 \pi} \Psi\left(v, \mathrm{~V}, \mathrm{~T}, \mathrm{O}, v^{\prime}, \mathrm{V}^{\prime}, \mathrm{S}\right) d v d \mathrm{~V} d \mathrm{~T} d \mathrm{~S} d \mathrm{O} \\
=\int_{0}^{\infty} \int_{0}^{\infty} \int_{0}^{\pi} \int_{0}^{\frac{\pi}{2}} \int_{0}^{2 \pi} \Psi\left(v^{\prime}, \mathrm{V}^{\prime}, \mathrm{T}^{\prime}, \mathrm{O}^{\prime}, v, \mathrm{~V}, \mathrm{~T}, \mathrm{O}, \mathrm{S}\right) \Delta d v d \mathrm{~V} d \mathrm{~T} d \mathrm{O} d \mathrm{~S} ; \\
\Delta=v^{2} \mathrm{~V}^{2} \tau: v^{\prime 2} \mathrm{~V}^{\prime 2} \tau^{\prime} .
\end{gathered}
$$

Therefore the third line of the equation (22) is also equal to $\int_{0}^{\infty} \int_{0}^{\infty} \int_{0}^{\pi} \int_{0}^{\frac{\pi}{2}} \int_{0}^{2 \pi} \delta^{2} l f^{\prime}\left(f \mathrm{~F}_{1}-f^{\prime} \mathrm{F}_{1}{ }^{\prime}\right) v^{2} \mathrm{~V}^{2} r \tau s \sigma d v d \mathrm{~V} d \mathrm{~T} d \mathrm{~S} d \mathrm{O}$; also the fourth is equal to $\int_{0}^{\infty} \int_{0}^{\infty} \int_{0}^{\pi} \int_{0}^{\frac{\pi}{2}} \int_{0}^{2 \pi} \delta^{2} l \mathrm{~F}_{1}{ }^{\prime}\left(f \mathrm{~F}_{1}-f^{\prime} \mathrm{F}_{1}{ }^{\prime}\right) v^{2} \mathrm{~V}^{2} r \tau s \sigma d v d \mathrm{~V} d \mathrm{~T} d \mathrm{~S} d \mathrm{O}$. For the sum of the third and fourth lines we find, by taking the arithmetic mean of the expressions found now and previously,

$\frac{1}{2} \int_{0}^{\infty} \int_{0}^{\infty} \int_{0}^{\pi} \int_{0}^{\frac{\pi}{2}} \int_{0}^{2 \pi} \delta^{2}\left(f^{\prime} \mathrm{F}_{1}^{\prime}-f \mathrm{~F}_{1}\right) l \frac{f \mathrm{~F}_{1}}{f^{\prime} \mathrm{F}_{1}^{\prime}} v^{2} \mathrm{~V}^{2} r \tau s \sigma d v d \mathrm{~V} d \mathrm{~T} d \mathrm{~S} d \mathrm{O}$.

The same transformations are to be applied to the first and second lines of equation (22). But in the latter expressions, moreover, the two impinging molecules play exactly the same part; so that we may here exchange the quantities which refer to the first molecule for those which refer to the second, and vice versâ. If both molecules belong to the same order, we have again, generally,

$$
\begin{aligned}
& \int_{0}^{\infty} \int_{0}^{\infty} \int_{0}^{\pi} \int_{0}^{\frac{\pi}{2}} \int_{0}^{2 \pi} \Psi\left(v, \mathrm{~V}, \mathrm{~T}, \mathrm{O}, v^{\prime}, \mathrm{V}^{\prime}, \mathrm{T}^{\prime}, \mathrm{O}^{\prime}, \mathrm{S}\right) d v d \mathrm{~V} d \mathrm{~T} d \mathrm{~S} d \mathrm{O} \\
= & \int_{0}^{\infty} \int_{0}^{\infty} \int_{0}^{\pi} \int_{0}^{\frac{\pi}{2}} \int_{0}^{2 \pi} \Psi\left(\mathrm{V}, v, \mathrm{~T}, \mathrm{O}, \mathrm{V}^{\prime}, v^{\prime}, \mathrm{T}^{\prime}, \mathrm{O}^{\prime}, \mathrm{S}\right) d v d \mathrm{~V} d \mathrm{~T} d \mathrm{~S} d \mathrm{O} .
\end{aligned}
$$

Therefore the first line of equation (22) may also be written

$$
\int_{0}^{\infty} \int_{0}^{\infty} \int_{0}^{\pi} \int_{0}^{\frac{\pi}{2}} \int_{0}^{2 \pi} \lambda^{2} l f_{1}\left(f^{\prime} f_{1}^{\prime}-f f_{1}\right) v^{2} \mathrm{~V}^{2} r \tau s \sigma d v d \mathrm{~V} d \mathrm{~T} d \mathrm{~S} d 0
$$

If we once more apply to this the transformation mentioned on the previous page, we obtain a fourth value for the first 
line of equation (22); and we have now to substitute the arithmetic mean of all four values. If we treat in the same way the second line of this equation, we obtain finally

$$
\begin{aligned}
& \frac{2}{\pi} \frac{d \mathrm{E}}{d t}=\int_{0}^{\infty} \int_{0}^{\infty} \int_{0}^{\pi} \int_{0}^{\frac{\pi}{2}} \int_{0}^{2 \pi} v^{2} \mathrm{~V}^{2} r \tau s \sigma d v d \mathrm{~V} d \mathrm{~T} d \mathrm{~S} d O \\
& \times\left[\lambda^{2}\left(f^{\prime} f_{1}^{\prime}-f f_{1}\right) \cdot l \frac{f^{\prime} f_{1}^{\prime}}{f f_{1}}+\Lambda^{2}\left(\mathrm{~F}^{\prime} \mathrm{F}_{1}^{\prime}-\mathrm{F} \mathrm{F}_{1}\right) \cdot l \frac{\mathrm{F}^{\prime} \mathrm{F}_{1}^{\prime}}{\mathrm{F}^{\prime} \mathrm{F}_{1}^{\prime}}\right. \\
& \left.+2 \delta^{2}\left(f^{\prime} \mathrm{F}_{1}^{\prime}-f \mathrm{~F}_{1}\right) \cdot l \frac{f \mathrm{~F}_{1}}{f^{\prime} \mathrm{F}_{1}^{\prime}}\right] .
\end{aligned}
$$

For the condition of equilibrium of energy, $f$ and $\mathrm{F}$ and also $\mathrm{E}$ must therefore be independent of the time; we must therefore have $\frac{d \mathrm{E}}{d t}=0$. But we see at once that the last-found integral for $\frac{d \mathrm{E}}{d t}$ represents a sum of infinitely small members, which are all negative or at most equal to zero; for if $f^{\prime} f_{1}^{\prime}-f f_{1}$ is positive the factor $l \frac{f f_{1}}{f^{\prime} f_{1}^{\prime}}$ is negative, and vice versâ.

Therefore $\frac{d \mathrm{E}}{d t}$ can only vanish when each of these members itself vanishes.

If the molecules of the first kind were very small in comparison with those of the second kind, then would $\lambda=0, \Lambda=\delta$. We may assume, still more generally, that the molecules of the first kind are perfectly permeable for each other, and so also those of the second kind for each other, and that each of the latter molecules only is surrounded by a sphere of radius $\delta$, at which the centres of the molecules of the first kind are reflected like infinitely small elastic spheres; in the latter case we should have $\Lambda=\lambda=0$. As soon as $\delta$ differs from zero, $f^{\prime} \mathrm{F}_{1}^{\prime}$ must always be equal to $f \mathrm{~F}_{1}$ for all values of the variables under the functional sign.

Since $v, \mathrm{~V}$, and $v^{\prime}$ are quite independent, and only $\mathrm{V}^{\prime}$ is determined by the equation of energy, we find, without difficulty,

$$
f=\mathrm{A} e^{-\hbar m v^{2}}, \quad \mathrm{~F}=\mathrm{B} e^{-h \mathrm{MV}^{2}} .
$$

But, in consequence of the impact of molecules of the first kind with those of the second, there will therefore be produced Maxwell's distribution of velocities and equality of mean energy amongst all the molecules. I take the present opportunity of remarking that I do not understand how gravitation 
is to be explained if we ascribe to Lesage's ultramundane particles the properties of gaseous molecules; for, whilst the sun protects the earth from certain impacts, it reflects other particles towards the earth which would not otherwise have reached it. Only, if the particles are not reflected by the earth and sun, but absorbed or perhaps reflected with a loss of energy, would attraction be produced between the sun and the earth.

I have carried out calculations similar to the above in my treatise, "Further Studies on the Thermal Equilibrium of Gaseous Molecules," * for a single kind of molecules after a somewhat different method, which, I believe, is characterized by great clearness; and I venture to recommend to the reader who is so disposed a perusal of the first section of this treatise.

We can easily apply the methor there described to a mixture of two kinds of gas. For the first of these let $\sqrt{ } \bar{x} \cdot \phi(x, t) d x$ be the number of molecules in the unit volume whose energy at the time $t$ lies between the limits $x$ and $x+d x$. Let $\phi(x, t)$ have a similar meaning for the second kind of gas. I have then used

$$
\sqrt{x \mathrm{X}} \phi(x, t) d x \phi(\mathrm{X}, t) d \mathrm{X} \psi\left(x, \mathrm{X}, x^{\prime}\right) d x^{\prime}
$$

to express the number of impacts which occur in unit time and volume between two molecules of the first kind, so that before impact their energies shall lie between the limits $x$ and $x+d x, \mathrm{X}$ and $\mathrm{X}+d \mathrm{X}$, whilst after impact that of the one molecule lies between $x^{\prime}$ and $x^{\prime}+d x^{\prime}$.

By variable distribution of conditions we are always to understand the number of impacts during a very short time divided by that time; $\psi$ has a similar meaning for the impacts of the molecules of the first kind with those of the second kind.

Then we easily convince ourselves that

$$
\begin{aligned}
& \sqrt{-\frac{\partial \phi(x, t)}{\partial t}}=\int_{0}^{\infty} \int_{0}^{x+\mathrm{x}} d \mathrm{X} d x^{\prime}\left[\phi\left(x^{\prime}, t\right) \phi\left(x+\mathrm{X}-x^{\prime}, t\right)\right. \\
& \sqrt{x^{\prime}\left(x+\mathrm{X}-x^{\prime}\right)} \psi\left(x^{\prime}, x+\mathrm{X}-x^{\prime}, x\right)-\phi(x, t) \phi(\mathrm{X}, t) \sqrt{x \mathrm{X}} \psi\left(x, \mathrm{X}, x^{\prime}\right) \\
& +\phi\left(x^{\prime}, t\right) \Phi\left(x+\mathrm{X}-x^{\prime}, t\right) \times \sqrt{x^{\prime}\left(x+\mathrm{X}-x^{\prime}\right)} \chi\left(x^{\prime}, x+\mathrm{X}-x^{\prime}, x\right) \\
& \left.-\phi(x, t) \Phi(\mathrm{X}, t) \sqrt{x \mathrm{X}} \chi\left(x, \mathrm{X}, x^{\prime}\right)\right]
\end{aligned}
$$

wherein we obtain $\sqrt{\mathrm{X}} \frac{\partial \Phi(\mathrm{X}, t)}{\partial t}$ by interchange of $\phi$ and $\Phi$,

- Sitzber. d. Wien. Akad. d. Wissensch. vol. lxvi., October 1872. 
$x$ and $\mathrm{X}, \psi$ and $\Psi$. In place of $x^{\prime}$ we have $\mathrm{X}^{\prime}$, the energy of the second molecule after impact.

We have further, except for a constant factor,

$$
\left.\begin{array}{rl}
\mathrm{E}=\int_{0}^{\infty} \sqrt{x} \phi(x, t)[l \phi(x, t)-1] d x & \\
& +\int_{0}^{\infty} \sqrt{\mathrm{X}} \Phi(\mathrm{X}, t)[l \Phi(\mathrm{X}, t)-1] d x, \\
\frac{d \mathrm{E}}{d t}=\int_{0}^{\infty} \sqrt{x} l \phi(x, t) \frac{\partial \phi(x, t)}{\partial t} d x+\int_{0}^{\infty} \sqrt{\mathrm{X}} l \Phi(\mathrm{X}, t) \frac{\partial \phi(\mathrm{X}, t)}{\partial t} d \mathrm{X},
\end{array}\right\}
$$

Before we substitute the above values in equation (24), we have still to establish two properties of the functions $\psi$, $\Psi$, and $\chi$. In the functions $\psi$ and $\Psi$ both impinging molecules play the same part. It is then just as probable that before impact the energy of the first should lie between $x$ and $x+d x$, that of the second beween $\mathrm{X}$ and $\mathrm{X}+d \mathrm{X}$, and, after impact that of the first between $x^{\prime}$ and $x^{\prime}+d x^{\prime}$, as that inversely before impact the energy of the first molecule should lie between $\mathrm{X}$ and $\mathrm{X}+d \mathrm{X}$, that of the second molecule between $x$ and $x+d x$, and after impact that of the second between $x^{\prime}$ and $x^{\prime}+d x^{\prime}$. Therefore that of the first lies between $x+\mathrm{X}-x^{\prime}$ and $x+\mathrm{X}-x^{\prime}+d x^{\prime}$. Or, in algebraic language,

$$
\begin{aligned}
& \Psi\left(x, \mathrm{X}, x^{\prime}\right)=\psi\left(\mathrm{X}, x, x+\mathrm{X}-x^{\prime}\right), . \quad . \\
& \Psi\left(\mathrm{X}, x, \mathrm{X}^{\prime}\right)=\Psi\left(x, \mathrm{X}, x+\mathrm{X}-\mathrm{X}^{\prime}\right) .
\end{aligned}
$$

The second property may be obtained as follows. We found the value (3) for the number $d Z$ of impacts which occur in unit time and volume between a molecule of the first and one of the second kind in such a way that the variables $v, \mathrm{~V}, \mathrm{~T}$, $\mathrm{S}, \mathrm{O}$ lie between the limits (2). We will first introduce the variables $r, G$ instead of $V$ and $T$. Then, instead of $G$, twice the energy of both molecules,

$$
y=(m+\mathrm{M}) v^{2}+\mathbf{M} r^{2}+2 \mathrm{M} v r g=(m+\mathrm{M}) v^{\prime 2}+\mathbf{M} r^{2}+2 \mathrm{M} v^{\prime} r g^{\prime} ;
$$

this gives

$$
d \mathrm{Z}=\frac{4 \pi^{2}}{\mathrm{M}} v r^{2} s \sigma d v d r d y d \mathrm{~S} d \mathrm{O} f(v) \mathrm{F}(\mathrm{V}) \delta^{2}
$$

We will now for the constants $v, r, y$, and $\mathrm{S}$ introduce the variable $v^{\prime}$ instead of $O$. Since in this $g$ is also constant,

$$
\begin{aligned}
& v^{2 l}=v^{2}+\frac{4 \mathrm{M} v r}{m+\mathrm{M}}\left(g \sigma^{2}-\gamma^{8 \sigma \sigma o}\right)+\frac{4 \mathrm{M}^{2} r^{2} \sigma^{2}}{(m+\mathrm{M})^{2}} \\
& d \mathrm{Z}=\frac{m+\mathrm{M}}{\mathrm{M}^{2}} \frac{2 \pi^{2} v^{\prime} r \delta^{2}}{\gamma \omega} d v d v^{\prime} d r d y d \mathrm{~S} f(v) \mathrm{F}(\mathrm{V}) .
\end{aligned}
$$

Phil. Mag. S. 5. Vol. 23. No. 143. April 1887. 
322 Prof. L. Boltzmann on the Assumptions necessary

If, lastly, instead of $v, v^{\prime}$ we introduce the energies $x$ and $x^{\prime}$,

$$
d \mathrm{Z}=\frac{m+\mathrm{M}}{m^{2} \mathrm{M}^{2}} \frac{2 \pi^{2} r \delta^{2}}{v \gamma \omega} d x d x^{\prime} d y d r d \mathrm{~S} f(v) \mathrm{F}(\mathrm{V}) .
$$

Now $4 \pi v^{2} f(v) d v=\sqrt{x} \phi(x, t) d x$; therefore

$$
f(v)=\frac{m \sqrt{m}}{4 \pi \sqrt{2}} \phi(x, t), \mathrm{F}(\mathrm{V})=\frac{\mathrm{M} \sqrt{\mathrm{M}}}{4 \pi \sqrt{2}} \Phi(\mathrm{X}, t) ;
$$

then, since for constants $x$ and $x^{\prime}$ evidently $d y=d \mathrm{X}$,

$$
d \mathrm{Z}=\frac{m+\mathrm{M}}{16 \sqrt{m \mathrm{M}}} \phi(x, t) \Phi(\mathrm{X}, t) \frac{r \delta^{2}}{v \gamma \omega} d x d x^{\prime} d \mathrm{X} d r d \mathrm{~S} .
$$

Hence the quantity formerly denoted by $\sqrt{x \overline{\mathrm{X}}} \boldsymbol{\chi}\left(x, \mathrm{X}, x^{\prime}\right)$ is equal to

$$
\frac{m+\mathrm{M}}{16 \sqrt{m \mathrm{M}}} \delta^{2} \iint \frac{r d r d \mathrm{~S}}{v \gamma \omega}
$$

where the integration is to be extended over all possible values with the given $x, \mathrm{X}, x^{\prime}$. If we interchange the values before and after impact, we have

$$
\sqrt{x^{\prime}\left(x+\mathrm{X}-x^{\prime}\right)} \chi\left(x^{\prime}, x+\mathrm{X}-x^{\prime}, x\right)=\frac{m+\mathrm{M}}{16 \sqrt{m \mathrm{M}}} \delta^{2} \iint \frac{r d r d \mathrm{~S}}{v^{\prime} \gamma^{\prime} \omega^{\prime}} .
$$

Since according to equation (16) $v^{\prime} \gamma^{\prime} \omega^{\prime}=v \gamma \omega$, and also the limits of the two double integrals are the same, it follows at once that

$$
\sqrt{x \overline{\mathrm{X}}} \chi\left(x, \mathrm{X}, x^{\prime}\right)=\sqrt{x^{\prime}\left(x-\mathrm{X}-x^{\prime}\right)} \chi\left(x^{\prime}, x+\mathrm{X}-x^{\prime}, x\right) \text {. }
$$

Two analogous equations hold for $\psi$ and $\Psi$.

The further calculations are now purely algebraical transformations of definite integrals, and are effected exactly as in the first section of the already mentioned "Further Studies." I will therefore only briefly indicate the method to be adopted. After substituting the values of $\frac{\partial \phi(x, t)}{\partial t}$ and $\frac{\partial \Phi(\mathrm{X}, t)}{\partial t}$ in equation (24), we have a term with

$$
l \phi(x, t) \cdot\left[\phi\left(x^{\prime}, t\right) \phi\left(x+\mathrm{X}+x^{\prime}, t\right)-\phi(x, t) \phi(\mathrm{X}, t)\right] .
$$

This, with the aid of an equation for $\psi$ analogous to equation (27), is to be transformed into a similar term, with the factor $-l \phi\left(x^{\prime}, t\right)$ before the square bracket. Both are, by means of equation (25), to be transformed into two terms, having the factors $l \phi(X, t)$ and $-l \phi\left(x+\mathrm{X}-x^{\prime}, t\right)$ before the 
square bracket. The arithmetic mean of the four expressions thus obtained is to be substituted in equation (24) for the transformed term. The term

$$
l \Phi(\mathrm{X}, t) \cdot\left[\Phi\left(\mathrm{X}^{\prime}, t\right) \Phi(x+\mathrm{X}-\mathrm{X}, t)-\Phi(\mathrm{X}, t) \Phi(x, t)\right.
$$

is to be treated in exactly the same way.

The remaining terms have now on ${ }^{1} y$ to be once transformed by means of equation (27), so that the factors $-l \phi\left(x^{\prime}, t\right)$ and $-l \Phi\left(x+\mathrm{X}-x^{\prime}, t\right)$ appear instead of the factors $l \phi(x, t)$ and $l \Phi(\mathrm{X}, t)$; and again the arithmetic mean of the original and transformed expressions is to be taken. In this way again $\partial \mathrm{E}$

$\frac{\partial \mathrm{L}}{d t}$ proves to be a sum of terms of which each vanishes separately if $\frac{d \mathrm{E}}{d t}$ vanishes. $\psi$ and $\Psi$ may be equal to zero. As soon as $\chi$ vanishes for no definite set of variables, that is only so soon as the molecules of the first kind impinge freely upon those of the second kind, these terms only vanish separately if Maxwell's distribution of velocities holds amongst the molecules of the first kind as well as those of the second kind.

The foregoing considerations serve also to completely establish the still more general proposition wiich Mr. Burbury has stated in the place already referred to. In fact, let us assume that the first kind of gas consists, as above, of very many molecules (molecules A) which do not impinge amongst themselves; but that the second kind, on the other hand, consists but of a single molecule $\mathrm{B}$ which comes into collision with the molecules of the first sort. Let the time of its free motion be great in comparison with the time of a collision. Let the whole be enclosed in a vessel $R$ with rigid elastic walls. Let $\mathrm{R}$ denote also the volume of the vessel. There must at length ensue a stationary condition in which the molecules of the first kind are, on the average, uniformly distributed through the vessel, and in which any direction is as probable as another fo: their velocity. In this condition, let there be in unit volume $4 \pi v^{2} f_{1}(v) d v$ molecules whose velocities lie between the limits $\left(b^{\circ}\right)$. The molecule $B$ will of course continually change its velocity; but if we take a very long time after the commencement of the stationary condition, then during that time its velocity, on the average, will, with equal probability, have assumed all possible directions in space; and the probability that its velocity will lie between the limits (7) will be expressed as some function of $V$, which we will denote by

$$
4 \pi \mathrm{V}^{2} \mathrm{RF}_{1}(\mathrm{~V}) d \mathrm{~V} \text {. }
$$

Let us now imagine a very great number of similar vessels 


\section{Prof. L. Boltzmann on the Assumptions necessary}

$R$, in each of which let there be an equal number of molecules A constituted as before, and each with the same distribution of velocities. Let $4 \pi v^{2} f(v) d v$ be the number of molecules in the unit volume whose velocities lie between the limits (6).

In each of the vessels $R$ let there be a single molecule $B$ which in each vessel shall be now here, now there, now moving in this direction, now in that with equal probability. Let the number of vessels $\mathrm{R}$ for which the velocity of the molecule $\mathrm{B}$ shall lie between the limits (7) be $4 \pi \mathrm{N} V^{2} R F(V) d V$, where $\mathrm{N}$ is the number of all the vessels, of which each has the volume R. Further, let there be no other impacts than those of the molecule $B$ in each vessel with the molecules $A$. Then ovidently, at least for the first moment of time, $\frac{\partial F}{\partial t}$ is determined by the same equation by which $\frac{\partial \mathrm{F}(\mathrm{V}, t)}{\partial t}$ was determined above, since it is quite indifferent whether all the molecules $B$ are in the same vessel or whether each is in a separate one.

But if we now imagine all the $N$ vessels of volume $R$ brought together into one, we obtain a vessel of volume NR in which in the unit volume there are $4 \pi \mathrm{V}^{2} \mathrm{~F}(\mathrm{~V}) d \mathrm{~V}$ molecules of the second kind whose velocities lie between the limits (7). In order that this may not be a proper fraction, but a very large number, we may imagine the unit volume to be very small in comparison with $\mathrm{R}$; that is, $\mathrm{R}$ as any large number, which must, however, be still very small in comparison with $\mathrm{N}$.

The change in $f$ will no doubt be different in the different vessels ; let us, however, denote by $f$ the arithmetic mean of all the values of $f$ for the different vessels; then again, at least in the first moment of time, $\frac{\partial f}{\partial t}$ will be given by the same equation as before, $\frac{\partial f(v, t)}{\partial t}$. In the former expressions we have of course $\lambda=\Lambda=0$, since neither the molecules $A$ impinge upon each other nor the molecules B. We convince ourselves most easily of the truth of the above assertion by imagining all the vessels $R$ united into one large vessel of volume $N R$, in which in the unit volume there are $4 \pi \mathrm{V}^{2} \mathrm{~F}(\mathrm{~V}) d \mathrm{~V}$ molecules $\mathrm{B}$ whose velocities lie between the limits (7), and $4 \pi f(v) v^{2} d v$ molecules $\mathrm{A}$ whose velocities lie within the linits (6). We may now suspect that in course of time $f$ assumes different values in different vessels, and not until the end becomes again equal in all. This suspicion is most easilv removed by supposing that at the beginning of the time $f$ and $\mathrm{F}$ have the values denoted above by $f_{1}$ and $F_{1}$. Everything then remains as it was; 
$\frac{\partial f}{\partial t}, \frac{\partial \mathrm{F}}{\partial t}$, and $\frac{d \mathrm{E}}{d t}$ will vanish. Nevertheless the former two magnitudes must be given by the equations (19) and (20) (where $\lambda=\Lambda=0$ ). Therefore also $\frac{d \mathrm{E}}{d t}$ must be given by the same equation (22) ; and, from the condition that it must vanish at the same time, it follows exactly as before that

$$
f=\mathrm{A} e^{-h m r^{2}}, \quad \mathrm{~F}=\mathrm{B} e^{-h \mathbf{M} \mathbf{L} 2},
$$

by which Mr. Burbury's proposition is proved. In this it is simply assumed that the number of molecules $\mathrm{A}$ is very large. This produces the effect that, so soon as the condition has become stationary, the distribution of velocities in each separate vessel is scarcely perceptibly influenced by the condition possessed by the molecule $B$ in that vessel.

It is only necessary to further remark here in passing, that the proof may be obtained in exactly the same way if, instead of regarding the molecules as elastic spheres, we assume any other law of mutual action ; if only, in the first place, the Lagrange-Hamilton equations of motion are applicable, and, in the second place, if the time of perceptible mutual action for each molecule is vanishingly small in comparison with the time of free motion.

\section{First Appendix.}

I have just received a treatise by $\mathrm{H}$. Stankewitsch*, which has for object to prove an equation which essentially is identical with equation (15) of this paper. I have long ago, in my treatise "Some General Propositions on the Equilibrium of Heat" $\uparrow$, called attention to the connection of a still more general equation with Jacobi's principle of the last multiplier. $\mathrm{H}$. Stankewitsch arrives at the proof of his equation in an altogether different way, which, however, is in every respect similar to Jacobi's proof of the principle of the last multiplier. However ingenions the method employed by Stankewitsch, I hope to show in the following lines that the equation in question may be proved much more simply in the way indicated by Maxwell. I will first show that the equation of $\mathrm{H}$. Stankewitsch is only an altered form of our equation (15). If $A$ be the angle between the velocity $v$ and the axis of abscissæ, $B$ the angle made by the $X Z$ plane with the plane which is parallel to the directions of $v$ and $O X$, and, lastly, $K$ the angle made by the last plane with the plane parallel to the

* Wiedemann's Annalen, Bd. xxix. p. 153 (1886).

$\dagger$ Wiener Sitzber. Bd. lviii. May 1871. 
directions of $v$ and $\mathrm{V}$; if, further, $\xi, \eta, \zeta$ be the components of $v ; \xi_{1}, n_{1}, \zeta_{1}$ those of $\mathrm{V}$ in the directions of the axes of coordinates: then

$$
\begin{aligned}
& d \xi d \eta d \zeta=v^{2} \alpha d v d \mathrm{~A} d \mathrm{~B}, \quad . \quad . \quad . \\
& d \xi_{1} d \eta_{1} d \zeta_{1}=\mathrm{V}^{2} \tau d \mathrm{~V} d \mathrm{~T} d \mathrm{~K} \text {. . . . . }
\end{aligned}
$$

If we denote the magnitudes with reference to the velocities $v^{\prime}$ and $\mathrm{V}^{\prime}$ after impact by a dash, we have

$$
\begin{aligned}
d \xi^{\prime} d \eta^{\prime} d \eta^{\prime} & =v^{2} \alpha^{\prime} d v^{\prime} d \mathrm{~A}^{\prime} d \mathrm{~B}^{\prime}, \ldots \\
d \xi_{1}^{\prime} d \eta_{1}^{\prime} d \zeta^{\prime}{ }_{1}=V^{\prime 2} \tau^{\prime} d \nabla^{\prime} d \mathrm{~T}^{\prime} d \mathrm{~K}^{\prime} \ldots & .
\end{aligned}
$$

Let us in fig. 2 denote the points of intersection of all these lines drawn from the centre $\Omega$ of a sphere of radius 1 with the surface of the sphere in the same way as the lines themselves. The variables $v$, V, T, S, O determine simply the magnitude and relative position of the lines determining the impact; they determine what I have called the form of the impact; $v^{\prime}, \mathrm{V}^{\prime}$, $\mathrm{T}^{\prime}, \Omega^{\prime}$ are therefore simple

Fig. 2.

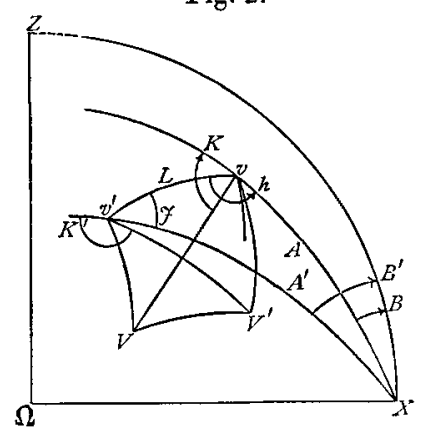
functions of the first-named variables. We will leave these variables constant, so that the whole form of the impact remains unaltered. Only its position in space, and so the variables $A, B$, and $K$ are to alter ; and the product of the corresponding changes in the variables $\mathrm{A}^{\prime}, \mathrm{B}^{\prime}, \mathrm{K}^{\prime}$, viz.

$$
d \mathrm{~A}^{\prime} d \mathrm{~B}^{\prime} d \mathrm{~K}^{\prime}=d \mathrm{~A} d \mathrm{~B} d \mathrm{~K} \cdot \Sigma \pm \frac{d \mathrm{~A}^{\prime}}{d \mathrm{~A}} \cdot \frac{d \mathrm{~B}^{\prime}}{d \mathrm{~B}} \cdot \frac{d \mathrm{~K}^{\prime}}{d \overline{\mathrm{K}}},
$$

is to be determined. It is geometrically evident that $d \mathrm{~A} d \mathrm{~B} d \mathrm{~K}$ must be equal to $d \mathrm{~A}^{\prime} d \mathrm{~B}^{\prime} d \mathrm{~K}^{\prime}$; for both sets of differentials may be supposed to be obtained by supposing that, for fixed position and magnitude of $v, v^{\prime}, \bar{V}, V^{\prime}$, the axis of abscissæ describes the whole interior of a cone of infinitely small aperture; and the system of coordinates revolves about the axis of abscissæ at a very small angle. This follows analytically in the following way. We see from fig. 2 that $\mathrm{B}^{\prime}=\mathrm{B}+\$ v \mathrm{X} v^{\prime}$. $\$ v \bar{X} v^{\prime}$ is simply a function of $A, K$, and the now constant angles. If, therefore, we now introduce $A^{\prime}, K^{\prime}, B^{\prime}$ instead of the variables $\mathrm{A}, \mathrm{K}, \mathrm{B}$, we have $d \mathrm{~B}^{\prime}=d \mathrm{~B}$. Therefore

$$
\Sigma \pm \frac{\partial A^{\prime}}{\partial A} \cdot \frac{\partial B^{\prime}}{\partial B} \cdot \frac{\partial K^{\prime}}{\partial K}=\Sigma \pm \frac{\partial A^{\prime}}{\partial A} \cdot \frac{\partial K^{\prime}}{\partial \bar{K}}
$$


In the latter functional determinant, besides the angles already put constant, B' is to be regarded as constant.

Further

whence

$$
\begin{aligned}
& a^{\prime}=a l+\alpha \lambda \cos h, \\
& \sin j: \sin h=\alpha: \alpha^{\prime} ;
\end{aligned}
$$

$$
\tan j=\frac{\alpha \sin h}{\sqrt{1-\alpha^{\prime 2}-\alpha^{2} \sin h}}=\frac{\alpha \sin h}{\alpha \lambda-\alpha l \cos h} .
$$

We see, further, from the figure that

$$
180^{\circ}-\mathrm{K}=h-\nless v^{\prime} v \mathrm{~V},
$$

when the latter angle depends simply on the form of the impact, and is therefore to be regarded at present as constant. So also

$$
j+180=\mathrm{K}^{\prime}+\Varangle \nabla^{\prime} v^{\prime} v .
$$

The latter angle again is constant; whence it follows, since nothing here depends upon the sign, that

$$
\Sigma \pm \frac{\partial A^{\prime}}{\partial A} \cdot \frac{\partial K^{\prime}}{\partial K}=\Sigma \pm \frac{\partial A^{\prime}}{\partial A} \cdot \frac{\partial j}{\partial h}
$$

Since in the equations for $\alpha^{\prime}$ and $\tan j$ also the angle $L$, which equally depends only on the form of the impact, plays the part of a constant, the determinant can be calculated without difficulty, and we obtain for it the value $\frac{\alpha^{\prime}}{\alpha}$. We might also have obtained this result without any calculation by imagining the points $v, v^{\prime}, V$, and $V^{\prime}$ as fixed. Since $A$ and $h$ are spherical polar coordinates of the point $X$ of the spherical surface, so also $\mathrm{A}^{\prime}, j$; the element of area ad Adh expressed by the former polar coordinates must be equal to the element of area $\alpha^{\prime} d A^{\prime} d j$ expressed by the latter. We have then

$$
\alpha^{\prime} d \mathrm{~A}^{\prime} d \mathrm{~B}^{\prime} d \mathrm{~K}^{\prime}=\alpha d \mathrm{~A} d \mathrm{~B} d \mathrm{~K} .
$$

For a fixed position of the points $v, v^{\prime}, \mathrm{V}$, and $\mathrm{V}^{\prime}, \mathrm{A}, \mathrm{K}$ and then $A^{\prime}, K^{\prime}$ may be regarded as spherical coordinates of the point $X$, which would give at once

$$
\alpha d \mathrm{~A}^{\prime} d \mathrm{~K}=\alpha^{\prime} d \mathrm{~A}^{\prime} d \mathrm{~K}^{\prime} \text {. }
$$

Since, further, from the definition of $\Delta$ (equation 13 ),

$$
d v^{\prime} d \mathrm{~V}^{\prime} d \mathrm{~T}^{\prime} d \mathrm{O}^{\prime}=\Delta d v d \mathrm{~V} d \mathrm{~T} d \mathrm{O}
$$

it follows from equations (28), (29), (30), (31) that

$$
\frac{d \xi^{\prime} d \eta^{\prime} d \zeta^{\prime} d \xi_{1}^{\prime} d \eta_{1}^{\prime} d \zeta_{1}^{\prime} d \mathrm{O}^{\prime}}{d \xi d \eta d \zeta d \xi_{1} d \eta_{1} d \zeta_{1} d \bar{O}}=\frac{v^{\prime 2} V^{\prime 2} \tau^{\prime} \Delta}{v^{2} V^{2} T}
$$


328 Prof. L. Boltzmann on the Assumptions necessary

Equation (15) is therefore proved by proving the equation

$$
d \xi^{\prime} d \eta^{\prime} d \xi^{\prime} d \xi_{1}^{\prime} d \eta_{1}{ }^{\prime} d \xi_{1}^{\prime} d 0^{\prime}=d \xi d \eta d \zeta d \xi_{1} d \eta_{1} d \zeta_{1} d \mathrm{O}, \text {. }
$$

and vice versû.

$O$ is here the angle between the planes $R \Omega R^{\prime}$ and $R \Omega v$ of fig. 1. If on the right-hand side of the equation (32) we introduce, instead of 0 , the angle $\psi$, which the former plane makes with the plane R $\Omega \mathrm{X}$ (compare fig. 3 ), $\xi, \eta, \zeta, \xi_{1}, \eta_{1}, \zeta_{1}$, and therefore also the angle between the planes $R \Omega X$ and $\mathrm{R} \Omega v$ remain constant; and since this is equal to the difference between $\Varangle 0$ and $\psi$, it follows that $d \psi=d O$. If in the same way we introduce upon the left-hand side of the equation (32) $\psi^{\prime}$ instead of $0^{\prime}$, it follows that

$$
d 0^{\prime}=d \psi^{\prime},
$$

and equation (32) becomes

$$
d \xi^{\prime} d \eta^{\prime} d \boldsymbol{\zeta}^{\prime} d \xi_{1}^{\prime} d \eta_{1}^{\prime} d \zeta_{1}^{\prime} d \boldsymbol{\psi}^{\prime}=d \xi d \eta d \zeta d \xi_{1} d \eta_{1} d \xi_{1} d \boldsymbol{\psi},
$$

which is exactly the form which H. Stankewitsch gives to the equation.

We will, however, further multiply each side by $\sigma d \mathbf{S}$, by which at the same time we indicate that $S$ is to be chosen as the eighth independent variable. The equation thus assumes the form

$d \xi^{\prime} d \eta^{\prime} d \zeta^{\prime} d \xi_{1}^{\prime} d \eta_{1}{ }^{\prime} d \xi_{1}^{\prime} d \psi^{\prime} \sigma d \mathbf{S}=d \xi d \eta d \zeta d \xi_{1} d \eta_{1} d \zeta_{1} d \psi \sigma d \mathbf{S}$.

We now again draw all the lines from the centre $\Omega$ of a sphere of unit radius, and denote in fig. 3 the points of intersection of the two relative velocities before and after impact with the surface of the sphere by $R$ and $R^{\prime}$; the ends of the two relative velocities by $R_{1}$ and $R_{1}{ }^{\prime}$. Let $H$ be the middle point of the arc $R R^{\prime}$ of a great circle, $X$ the point in which the axis of abscissa intersects the surface of the sphere. We now for constants $\xi, \eta, \zeta, \xi_{1}, \eta_{1}, \zeta_{1}$ introduce the

Fig. 3.

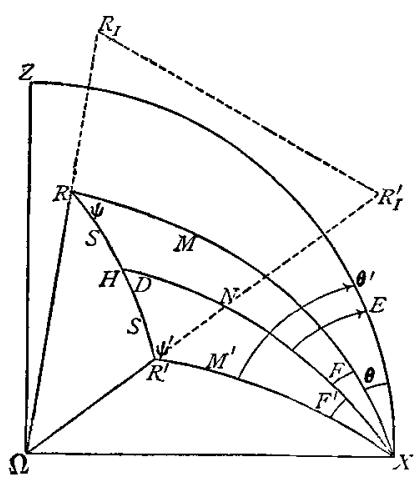
angles $\nless N=X H$ and $E=Z X H$ instead of $\nless S=R H$ and $\psi=\mathrm{XRR}^{\prime}$. Since, again, for a fixed position of the points $X, Z$, and $R$, both ' $S$ and $\psi$ as well as $N$ and $E$ are spherical 
polar coordinates of the point $H$ of the sphere, we have

$$
v d \mathrm{~N} d \mathrm{E}=\sigma d \mathrm{~S} d \psi \text {. }
$$

The left side of equation (33) is next transformed into

$$
d \xi d \eta d \zeta d \xi_{1} d \eta_{1} d \zeta_{1} v d \mathrm{~N} d \mathrm{E} . \quad . \quad . \quad .
$$

If, now, we denote the projections of the relative velocity $\Omega \mathrm{R}_{1}$ before impact on the axes of coordinates by $x, y, z$, and also the projections of the relative velocity $\Omega_{R_{1}}{ }^{\prime}$ after impact on the axes of coordinates by $x^{\prime}, y^{\prime}, z^{\prime}$, and with constant $\xi, \eta, \zeta$ introduce the variables

expression (34) becomes

$$
x=\xi_{1}-\xi, \quad y=\eta_{1}-\eta, \quad z=\zeta_{1}-\zeta,
$$

$$
d \xi d \eta d \zeta d x d y d z \nu d \mathrm{~N} d \mathrm{E} \text {. }
$$

Then we leave $x, y, z, \mathrm{~N}, \mathrm{E}$ constant, and instead of $\xi, \eta, \zeta$ introduce the variables $\xi^{\prime}, \eta^{\prime}, \zeta^{\prime}$. If $x, y, z$ be the projections of the line $R_{1} R_{1}{ }^{\prime}$ of the relative velocities drawn from $\Omega$ on the axes of coordinates, we have

$$
\xi^{\prime}=\xi-\frac{\mathrm{M} x_{1}}{m+\mathrm{M}} ; \quad \eta^{\prime}=\eta-\frac{\mathrm{M} y_{1}}{m+\mathrm{M}} ; \quad \zeta^{\prime}=\zeta-\frac{\mathrm{M} \tilde{z}_{1}}{m+\mathrm{M}} .
$$

Since, now, all the lines drawn in fig. 3 remain altogether unaltered in magnitude and position, $x_{1}, y_{1}$, and $z_{1}$ are also constant, and we have

$$
d \xi^{\prime} d \eta^{\prime} d \xi^{\prime \prime}=d \xi d \eta d \xi^{\prime}
$$

Hence expression (35) becomes

$$
d \xi^{\prime} d \eta^{\prime} d \zeta^{\prime} d x d y d z \nu d \mathrm{~N} d \mathrm{E} . \quad \text {. . . . }
$$

The next step consists in introducing for constant $\xi^{\prime}, \eta^{\prime}, \zeta^{\prime}$, $\mathrm{N}, \mathrm{E}$ the variables $x^{\prime}, y^{\prime}, z^{\prime}$ instead of $x, y, z$; that is, the coordinates of the point $R_{1}{ }^{\prime}$ instead of the coordinates of the point $R_{1}$. It is at once seen from fig. 3 that the element of volume described by the point $R_{1}$ on change of its coordinates is exactly equal to that which the point $R_{1}^{\prime}$ describes for the position of the point $H$ remains unchanged. It follows, therefore, that

$$
d x d y d z=d x^{\prime} d y^{\prime} d z^{\prime} ; \quad \text {. . . . . }
$$

and expression (36) becomes

$$
d \xi^{\prime} d \eta^{\prime} d \zeta^{\prime} d x^{\prime} d y^{\prime} d z^{\prime}, \nu . d \mathrm{~N} . d \mathrm{E} . \quad \text {. . . }
$$

Now, again, inversely

$$
\xi_{1}^{\prime}=\xi^{\prime}+x^{\prime}, \quad \eta_{1}^{\prime}=\eta^{\prime}+y^{\prime}, \quad \zeta_{1}^{\prime}+\xi^{\prime}+z^{\prime}
$$


330 Prof. L. Boltzmann on the Assumptions necessary are introduced instead of $x^{\prime}, y^{\prime}, z^{\prime}$, so that the expression (38) becomes

$$
d \xi^{\prime} d \eta^{\prime} d \xi^{\prime} d \xi_{1}^{\prime} d \eta_{1}^{\prime} d \xi_{1}^{\prime} v d \mathrm{~N} d \mathrm{E}
$$

Lastly, we introduce, instead of the spherical polar coordinates $\mathrm{N}, \mathrm{E}$ of the point $\mathrm{H}$, its spherical polar coordinates $\mathrm{S}, \psi^{\prime}$; so that we obtain

$$
\nu d \mathrm{~N} . d \mathrm{E}=\sigma d \mathrm{~S} . d \psi^{\prime} .
$$

Lastly, expression (30) becomes therefore

$$
d \xi^{\prime} d \eta^{\prime} d \zeta^{\prime} d \xi_{1}^{\prime} d \eta_{1}^{\prime} d \zeta_{1}^{\prime} \sigma d \mathrm{~S} . d \psi^{\prime},
$$

by which equation (33) is proved.

If we prefer to prove equation (37) analytically, fig. 3 would give

$$
x=r m, \quad y=r \mu \sin \theta, \quad z=r \mu \cos \theta,
$$

where $\Omega R_{1}=\Omega R_{1}^{\prime}=r$.

$$
\begin{aligned}
& \delta: \phi=\mu: \sigma, \quad \delta: \phi^{\prime}=\mu^{\prime}: \sigma, \quad \mu^{\prime} \phi^{\prime}=\mu \phi, \\
& s=m n+\mu \nu f, \quad \sigma^{2} d^{2}=\sigma^{2}-\mu^{2} \phi^{2} \\
& \quad=1-(m n+\mu \nu f)^{2}-\mu^{2} \phi^{2}=(m \nu-\mu n f)^{2}, \\
& m^{\prime}=n s+\nu \sigma d=m n_{2}+\mu \nu_{2} f,
\end{aligned}
$$

where

From

$$
n_{2}=\cos 2 \mathrm{~N}, \quad \nu_{2}=\sin 2 \mathrm{~N} \text {. }
$$

it follows that

$$
s=m^{\prime} n+\mu^{\prime} \nu f^{\prime}=m n+\mu \nu f,
$$

$$
\mu^{\prime} f^{\prime}=m \nu_{2}-\mu f n_{2} \text {. }
$$

If in this equation and in the equation $\mu^{\prime} \phi^{\prime}=\mu \phi$ we put

$$
f^{\prime}=e \cos \theta^{\prime}+\epsilon \sin \theta^{\prime}, \quad \phi^{\prime}=e \sin \theta^{\prime}-\epsilon \cos \theta^{\prime},
$$

it follows that

$$
\begin{aligned}
& \mu^{\prime} \cos \theta^{\prime}=m e \nu_{2}-\mu e f n_{2}-\mu \epsilon \phi, \\
& \mu^{\prime} \sin \theta^{\prime}=m \epsilon \nu_{2}-\mu \epsilon f n_{2}+\mu e \phi .
\end{aligned}
$$

By multiplying by $r$ and observing that

$$
\begin{gathered}
r m^{\prime}=x^{\prime}, \quad r \mu^{\prime} \sin \theta^{\prime}=y^{\prime}, \quad r \mu^{\prime} \cos \theta^{\prime}=z^{\prime}, \\
f=e \cos \theta+\epsilon \sin \theta, \quad \phi=\epsilon \cos \theta-e \sin \theta, \\
r \mu f=\epsilon y+e z, \quad r \mu \phi=-e y+\epsilon z,
\end{gathered}
$$

we obtain

$$
\begin{aligned}
& x^{\prime}=n_{2} x+\nu_{2} \epsilon y+\nu_{2} e z, \\
& y^{\prime}=\nu_{2} \epsilon x-\left(e^{2}+\epsilon^{2} n_{2}\right) y+2 \nu^{2} e \epsilon z, \\
& z^{\prime}=\nu_{2} e x+2 \nu^{2} e \epsilon y-\left(\epsilon^{2}+e^{2} n_{2}\right) z,
\end{aligned}
$$


for the Theoretical Proof of Avogadro's Law.

and we can then convince ourselves directly that

$$
\Sigma \pm \frac{\partial x^{\prime}}{\partial x} \cdot \frac{\partial y^{\prime}}{\partial y} \cdot \frac{\partial z^{\prime}}{\partial z}=1
$$

Although I have already deduced a great variety of relations from fig. 1, yet it would probably furnish several other equations which might be of use in particular circumstances, e. $g$. by denoting the magnitude and position of the straight lines $v, \mathrm{~V}, v^{\prime}, \mathrm{V}^{\prime}$ symmetrically by the magnitude and position of the straight lines $\Omega, P$ and of the line joining the point $P$ with the middle point of the straight line $W^{\prime}$. Symmetrical relationships of this kind are particularly convenient when we wish to obtain equations in which the magnitudes before and after impact play the same part as the equation we have used.

$$
\sqrt{x \overline{\mathrm{X}}} \cdot \chi\left(x, \mathrm{X}, x^{\prime}\right)=\sqrt{x^{\prime}\left(x+\mathrm{X}-x^{\prime}\right)} \cdot \chi\left(x^{\prime}, x+\mathrm{X}-x^{\prime}, x\right) .
$$

\section{Second Appendix.}

After correcting the foregoing for the press, I became acquainted, by the kindness of the author, with Prof. Tait's paper "On the Foundations of the Kinetic Theory of Gases"*. While reserving for a future occasion my remarks on Prof. Tait's observations on the mean path, and on the case when external forces act, I will here mention only one point. If in a gas on which no external forces act, and whose molecules are elastic spheres, $\mathrm{F}(x, y, z) d x d y d z$ be the probability that components of the velocity of a molecule parallel to the axes of coordinates shall at the same time lie between the limits $x$ and $x+d x, y$ and $y+d y, z$ and $z+d z$, then Maxwell bases the first proof which he gives $\dagger$ of his law of distribution of velocities on the assumption that $\mathrm{F}(x, y, z)$ is a product of these functions, of which the first contains only $x$, the second only $y$, the third only $z$. This is the same as the assumption that, for a given component of velocity at right angles to the axis of abscissæ, the quotient of two probabilities, viz. the probability that the component of the velocity of a molecule in the direction of the axis of abscissæ lies between $x$ and $x+d x$, and the probability that the same quantity lies between certain other limits $\xi$ and $\xi+d \xi$, is altogether independent of the given value of the component of the velocity of the same molecule at right angles to the axis of abscissæ. In a

* Trans. Ruy. Soc. Edin. xxiii. p. 65 (1886).

† Phil. Mag. [4] rol. xix. p. 19 (1860). 
later paper* Maxwell himself speaks of this assumption as precarious; and therefore gives a proof resting on a quite different foundation. In fact, we should expect that greater velocities in the direction of the axis of abscissæ in comparison with the smaller ones would be so much the more improbable the greater the component of velocity of the molecule at right angles to the axis of abscissæ. If, for example,

$$
\mathrm{F}(x, y, z)=c e^{-h\left(x^{2}+y^{2}+z^{2}\right)^{2}}=c e^{-h\left(y^{2}+z^{2}\right) 2} \cdot e^{-h\left[x^{4}+2 x^{2}\left(y^{2}+z^{2}\right)\right]} ;
$$

then the quotient just mentioned would be

$$
\frac{\mathbf{F}(x, y, z) d x}{\mathbf{F}(\xi, y, z) d \xi}=\frac{d x}{d \xi} \cdot e^{k\left(\xi^{4}-x^{4}\right)} \cdot e^{2 h\left(\xi^{2}-x^{2}\right)\left(y^{2}+x^{2}\right)} .
$$

The larger $\sqrt{y^{2}+z^{2}}$, the more would small values in comparison with large ones gain in probability. Now, by means of the law of distribution of velocities, which is to be proved, we obtain the proof of the very remarkable theorem : that the relative probability of the different values of $x$ is altogether independent of the value, supposed to be given, which $\sqrt{y^{2}+z^{2}}$ has for the same molecule; that therefore the quotient $\mathrm{F}(x, y, z)$ : $\mathrm{F}(\xi, y, z)$ is independent of $y$ and $z$; or, what is the same, since the three axes of coordinates must play the same part, that $\mathrm{F},(x, y, z)$ may be represented as a product of three functions of which the first contains only $x$, the second only $y$, the third only $z$.

It is therefore an altogether inadmissible circulus viliosus to make use of this assumption to prove Maxwell's law of distribution of velocities. This therefore also holds good of the proof which Prof. Tait has given (pp. $68 \& 69$ of the paper quoted), and which is only a reproduction of Maxwell's first proof, which he himself later rejected. For, from the circumstance that the distribution of velocities must be independent of the special system of coordinates chosen for its calculation, we can never show that $\mathrm{F}(x, y, z)$ must have the form $f(x) \phi(y) \psi(z)$, only when this has been already proved. One might make use of the circumstance to show the similarity of form of the three functions $f, \phi$, and $\psi$. I do not even need to enter upon known geometrical investigations if the value of a function of three rectangular coordinates $x, y, z$ is independent of the choice of the system of coordinates. For Prof. Tait has already shown of the function denoted above by $F$, that it can only be a function of $\sqrt{x^{2}+y^{2}}+z^{2}$; but the value of the expression $\sqrt{x^{2}+y^{2}+z^{2}}$ is already quite independent of the special posi-

$$
\text { * Phil. Mag. [4] xxxv.p. } 145 \text { (1868). }
$$


Arc-Lamp suitable for use with the Duboscq Lantern. 333 tion of the system of coordinates ; therefore evidently any function whatever of $\sqrt{x^{2}+y^{2}} \overline{-} z^{2}$ fulfils the same condition, and by this condition no other further property of the function $\mathrm{F}$ can be disclosed. As, for example, the value of the above-used function $e^{-h\left(x^{2}+y^{2}+z^{2}\right)^{2}}$ is also entirely independent of the special choice of the system of the coordinates, although it does not permit of being reduced to the form $f(x), \phi(y)$, $\psi(z)$.

XXXVII. On an Arc-Lamp suitable to be used with the Duboseq Lantern. By. Professor Silvanus P. Thompson, D.Sc.*

[Plate III.]

THE lamp devised by Foucault and Duboscq, and supplied for so many years by the famous house of Duboscq, fails to fulfil the electrical requirements of the modern physical laboratory, though it has rendered excellent service in the past. Yet the lantern and optical adjuncts of the standard pattern of Duboscq are so widely used that it seemed desirable to find some other arc lamp which, while fulfilling the electrical requirements of the case, could be used with the Duboseq lantern.

Before describing the lamp which I have for twelve months employed for this purpose, I propose to state the conditions to be fulfilled, and the reasons why the old Duboseq lamp fails to fulfil them.

The modern physical laboratory is usually supplied with electric energy under one of two alternative conditions, namely either at constant potential or with constant current; more usually under the former condition. If supplied from a dynamo the dynamo may be either series-wound, shuntwound, or compound-wound. If supplied from accumulators the accumulators will work at constant potential, and will have a very small internal resistance.

The arc-lamp for laboratory use must be capable of working under the given conditions. No doubt the Duboseq lamp worked fairly when supplied with current from 50 Grove's cells. But in a laboratory where there is another and better and less wasteful source of supply, 50 Grove's cells are not desirable. Though 40 accumulators have an electromotive force almost exactly equal to that of 50 Grove's cells, the Duboscq lamp does not work well with them unless a resistance of several ohms is intercalated in the circuit to represent

* Communicated by the Physical Society. 